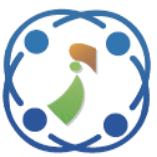

\title{
Multilingual Sentiment Analysis Using the Social Eagle-Based Bidirectional Long Short-Term Memory
}

\author{
Deepali Londhe ${ }^{1 *}$ \\ Aruna Kumari ${ }^{2}$ \\ ${ }^{1}$ Computer Science and Engineering Department, K.L.University, Vijaywada, Andra Pradesh, India \\ ${ }^{2}$ Computer Science and Engineering Department VJIT, Hydrabad, Ph.D. Guide at Computer Science and \\ Engineering Department, K.L. University, Vijaywada, Andhra.Pradesh.,India \\ * Corresponding author’s Email: deep.londhe@gmail.com
}

\begin{abstract}
Rapid evolvement in the field of social media constitutes an increasing demand in sentiment prediction for effective communication. Sentimental analyses are employed to extract opinions from individuals in order to invigorate the quality of material or a product for the effective growth of an organization. Researchers are aspired to predict the sentiment through comments of different languages, but there is a lack in the dictionaries accessible for different languages. In this research, hybridized Social Eagle Algorithm-based deep bidirectional long short-term memory (SoEo Algorithm-based deep BiLSTM) is proposed to predict the sentiment effectively. The languages are identified and converted into a standard format by the process of Transliteration and the features are extracted from these standardized data. The hunting strategy of the bald eagle and the adaptation behavior of coyotes are hybridized and executed in both forward and backward directions utilizing the BiLSTM classifier. The simulation outcome shows that the proposed model obtained an accuracy of $91.572 \%$, precision of $89.196 \%$, recall of $91.551 \%$ and F1 measure of $89.019 \%$, which will be more efficient compared to the state-of-art methods.
\end{abstract}

Keywords: BiLSTM classifier, Transliteration, SoEo algorithm, Deep BiLSTM, Sentiment analysis.

\section{Introduction}

The living standard of human life has been changing due to the evolution of World Wide Web, which reflects in the thinking capacity of the individual. In the days of yore, the decisions were made through confessing their problems with friends, family members or relatives. On contrary, the business decisions were made through polling. Nowa-days, due to the emanation of technologies, like forum, blogs, reviews and so forth, the process of decision-making has been changed for both individuals and business decisions. Thus, the extraction or computation of opinions is termed as sentiment analysis [1]. Coming to the study of sentiment analysis, the data that are collected from the unstructured texts are converted into structured information based on the information, such as opinion, attitude, or emotions of a particular individual [2,3]. The polarity or the degree of importance can be extracted from the opinions, emotion, attitudes, and similar sentiments collected and created as a document based on positive, negative, or neutral [4]. Hence, the sentiment analysis is also referred as opinion mining. Therefore, sentiment analysis and affective classification gains significance as the individual opinion for a product or event is understood, which promotes the business industry for an effective marketing, political campaigns and helps to understand the public relations [5-7].

At present, the sentiment analysis uses the single language particularly, English, but due to a rapid increase in the usage of Internet, a massive growth is reported in the social media applications, such as Facebook, Twitter, Instagram and so forth the people adapt themselves to their mother tongue for posting and messaging, which insists a necessity for handling the multiple language-based reviews [8]. For example: In AirBnB, Amazon and TripAdvisor, there 
are around 400 billion of people, using these networks in a month. Thus, the sentiment analysis for the multi-lingual data is the need of an hour. Accounting this scenario, the distribution is unbalanced for different languages, with fewer reviews for some languages, resulting in the shortfall of the data in such a way that the existing sentiment analysis algorithms struggle to yield good results. Due to this inefficiency, the sentiment analysis algorithms depend on the languages with greater density of reviews, which leads to an increased risk of missing essential information in texts written in other languages [8]. Thus, the innovation of multilingual sentiment analysis techniques using multiple languages for the sentimental analysis by analyzing the data in different languages is more efficient when compared with the circumstances of using a single language [3, 8, 9].

The major challenging task in language specific methods for opinion mining is that there are a wide variety of languages namely, Arabic, Chinese, French, German, Hindi, Italian, Japanese, Russian, Spanish and Thai, but there is a lack in sentiment dictionaries excluding English [10]. At present, research are proceeding on the automatic or semi-automatic generation of Large non-English resources for performing sentimental analysis to resolve this problem [11, 12]. For availing multiple languages, SentiStrength lexical resources [13] are made available for automatically translating those languages excluding some languages, such as Spanish because the resources were later improved by capitalizing additional lexica, which gains an overall improvement over foreign language $[14,15]$. Ghorbel and Jacot [16] made an effort to translate English SentiWordNet entries into French and concluded that two parallel words always have individual semantic orientation even though if the translation is correct due to the difference in common usage [12]. The unique advantages in solving small sample, non-linear and high dimensional pattern recognition in machine learning insists us to make use for classification purposes and therefore BiLSTM methods are utilized for effective emotion recognition.

The research concentrates on developing a SoEo Algorithm-based deep BiLSTM classifier that concentrates on sentiment classification through the multilingual input data. Initially the data are collected and are classified based on the slang or an emotion and the words are converted into a standardized format utilizing transliteration process. A BiLSTM classifier is employed in this research for the effective classification of the sentiments from the multi-lingual tweets or reviews. The language of the conversation is identified and the slang words, emojis, and so on, in the reviews are converted into the standard presentation in the language identification and the conversion phase, which enables the easy identification of sentiments from the tweets. The main highlight of the research lies in the tuning of hyper-parameters of the classifier using the proposed SoEo algorithm. The major contribution of the research:

SoEo Algorithm - A meta-heuristic algorithm: The SoEo algorithm is a meta heuristic swarm intelligence algorithm which is developed by the hybridization of the social condition and experience characteristics of coyote with descending characteristics of Eagle to prey in an effective manner.

SoEo-based Deep BiLSTM classifier: Sentiment classification is performed using SoEo-based deep BiLSTM classifier, where the proposed SoEo algorithm is used to tune the hyperparameters of deep BiLSTM classifier, for boosting the classification accuracy.

The organization of the manuscript follows: the review of the literature with the need for the sentiment classification model is enumerated in Section 2. The need of the proposed SoEo Algorithmbased deep BiLSTM, algorithmic procedure and steps are detailed in Section 3. The analysis of the proposed sentiment classification model is elucidated in Section 4 and the conclusion is deliberated in Section 5.

\section{Motivation}

All In this section, the motivation behind the research is enumerated. The existing methods are concentrating mainly on single language processing and the challenges associated with the single language processing motivated to research on the multilingual sentiment analysis.

\subsection{Literature survey}

In recent years, the sentiment analysis plays an important role, but the contributions are always based on the English language. To make it more efficient R.Bhargava et al. [1] conveyed that by analyzing the text present in the reviews using multilingual sentimental analysis through text summarizing in machine learning, the information is utilized from less number of sentences, but the drawback is that regional languages are not included.

Wehrmann et al. [17] developed a Language agnostic translation free method for Twitter sentiment analysis by implementing deep convolutional neural networks with character level embeddings for determining the polarity found in 
different languages and it provided results of high accuracy with learnable parameters, but it requires a large dataset for training.

Diverging from other old techniques Jain et al. [18] Created an advanced framework for the detection of emotions in multilanguage text data using emotion theories which deals with psychology and linguistics utilizing Intelligent text processing and computational linguistics and it is highly reliable for Computer human interaction, but the data like emoticons are not generally considered.

Vilareset et al. [12] introduced an easy and replicable method named BabelSentiNet for automatically generating the SentiNet using statistical machine translation tools by creating a high coverage Sentinet version of the target language which is low in cost and high in speed, but when it comes to multiword the detection should be improved.

Tianrong Rao et al.[19] aimed to utilize different levels of visual features from both global and local point of view by discovering the sentimental response of local regions using multi-level region-based Convolutional Neural Network. In the perspective of both local and global level, it works more efficiently, and it fails to recognize multiple emotions at the same time.

Enduring technology of Deep Neural Network (DNN) motivated Linhui Sun et al.[20] to develop the DNN decision tree SVM model which can excavate deep emotion information as well as distinctive emotion features from easily confused emotions and it attains higher recognition rate, but it fails to find more distinctive features.

Mario Graff et al. [6] contributed an SA System named EvoMSA which makes us unique from others while participating in various SA competitions through their characteristics such as domain independent and multilingual using language independent techniques by processing texts. The system has significant potential power to recognize multiple languages, but the sentence polarity prediction may skip some important information carried-on by the emoticons.

Madani et al. [8] approached a new hybrid approach depending on the semantic similarity using the WordNet dictionary and the fuzzy logic with three important steps is as follows the fuzzification, the rule inference or aggregations and the defuzzification for classifying the tweets as positive, negative or neutral and this technique familiarizes the fuzzy logic and Hadoop framework for obtaining the opinion documents which work more efficient, accurate, recall, precision but the classification networks employed were ineffective, degrading the performance.
Zera et al. [21] experimented and highlighted the ensemble learning effect using a majority voting technique for cross-corpus, multilingual speech emotion recognition system and it tackles the emoticons wisely, which leads to the global utilization of robots that is a complex process for detecting the emoticons from the sentiment. The major drawback of the method was that the recognition accuracy is less due to the poor performance of the classifier.

\subsection{Challenges}

The detailed need for the proposed sentiment prediction model is discussed below. It is a difficult task to extricate the text information from the conventional multi-lingual sentiment analysis using most of the classifiers.

In conventional multi-lingual emotion recognition methods, the complexity of the process increases when it comes to the identification of emojis and slang words.

Performance of the emotion recognition system is degraded when classification of the process is not involved in most of the cases.

The stumbling block of the emotion recognition system is the high execution time due to the complicated training process.

A lot of prediction models based on machine learning and deep learning are used, which suffer from the vanishing gradient issues and long-term information preservation act as the burden in existing multi-lingual sentiment analysis. Hence, this challenge is addressed through the usage of the optimized deep LSTM classifier.

The major shortcoming of the existing methods relies in their inability to detect the sentence polarity from the multi-language chats particularly, in dealing with the emojis, slang words and so on. In this research, we aim at designing a sentiment prediction model from the multi-lingual conversation that includes the slang words, emojis, abbreviations, and so on.

\subsection{Proposed sentiment prediction model using the proposed hybrid optimization dependent BiLSTM classifier}

This research aims at developing a multilingual sentiment analysis model based on the key aspects of enlarged users in the social media, and to conquer the disadvantages of the single language analysis. Moreover, multilingual sentiment analysis allows us to extract the emotions from diverse users using different comments that accompany us to classify 

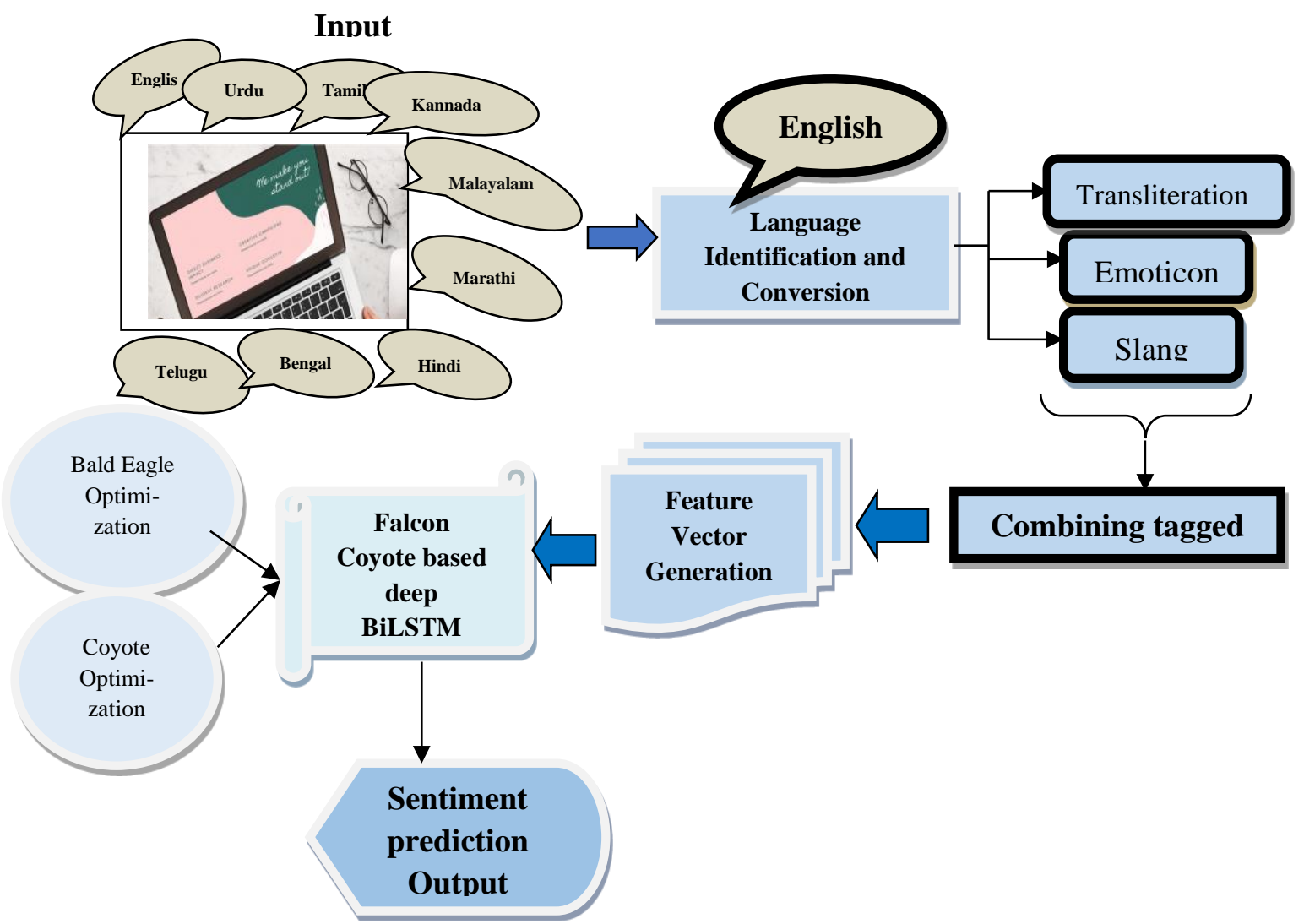

Figure. 1 Representation of sentiment prediction model

based on the sentimental polarity, such as positive and negative. Following the multilingual analysis [22], the initial step is to collect the input data from the comments or tweets provided by the user in the social media [23]. The raw data [24, 25] consists of the emojis, slang words, abbreviation, or any other tilt words, which are recognized, filtered, and converted into a standardized tagged data using the transliteration process. The features are extracted from the tagged data, which is then subjected to the sentiment classification using the SoEo algorithm based deep BiLSTM Classifier. Fig. 1 shows the block diagram for the proposed model of sentiment analysis from the multi-lingual data.

\section{Method}

\subsection{Language identification and conversion}

i) Language Identification: In the multi-lingual environment, the substantial challenge is to identify the language for which initially, the language is recognized from the sentence, which is subjected to translation for identifying the sentiment as either positive or negative. There are several approaches for language identification, which includes the character analysis, short word approach, training using classifier, and dictionary-based approach. Trigrams and short word approach are also prominent techniques to identify language. It is peculiar to note that different languages possess different character sets, which supports the review process towards language identification. Though the techniques, like short-word approach, trigrams, dictionaries, and the classifiers, like naïve bayes are employed for language identification, code mixing between inter and intra-sentences and words is a challenge particularly, for any language encoder.

In this research, language detection library in Python named langdetect-1.0.9 version is used for language identification, which supports 55 languages.

ii) Data Normalization: The data normalization is performed on the multilingual data to process the abbreviations, emoticons, slang words, and so on. The informal words from the conversations are identified and converted into a standard format in order to effectively classify the sentiment.

iii) Emotions: People can express their feeling through emoticons since it looks to be more attractive, colorful, inexpensive and expressive especially for teens. Individuals use emoticons to emphasize their feelings. The correct decoding of emoticons in sentimental analysis helps us to improve the accuracy rate of sentiment prediction. In this research, the emoticons are transformed to a standard form using the python library "emoji 1.5.0". 
iv) Slang word: Slang words are defined based on the culture, trend or a locality and the slang words are identified using the Slang dictionary. v) Transliteration: The texts that are obtained from the data normalization and language identification are subjected to the transliteration phase depending on the availability of the language resources and the sentimental analysis approaches employed for classification. Transliteration allows the conversion to the original script in order to classify the sentiment associated with the texts, generated from a sentence or conversation.

\subsection{Establishment of the feature vector}

The feature vector is established using the feature extraction, which boosts the classification performance through the presentation of the significant information from the input dataset. The polarity of the sentiment is classified based on the extracted features and in the case of multiple positive and negative words, it is difficult to assign equal importance to all words. To conquer this, the special words are selected based on the relevant part of the speech defined by the adjective, adverb or a verb that seems to be more effective in terms of the emotions. The features extracted from the input data is based on the TF-IDF feature, which defines the frequency of the meaningful words in the conversation.

\subsubsection{Term frequency-inverse document frequency (TF-IDF) features}

The TF-IDF feature is a common measure used for assigning importance to a word in the conversation and the measure is a combination of TF and IDF as given by,

$$
\operatorname{IDF}(r, a)=\frac{\log [(|z|+1)}{D F(r, z)+1}
$$

where, $r$ represents a word and $a$ represents the sentence of a comment. $(T F(r, a))$ represents the frequency of the word $r$ in the sentence $a$ and $D F(r, z)$ is the number of sentences that contains the word $r .|z|$ represents the total number of sentences present in the comments.

$$
T F-I D F(r, a, z)=T F(r, a) \cdot \operatorname{IDF}(r, z)
$$

TF-IDF is simply represented by the amalgamation of the product of TF and IDF features. The dimension of the feature vector is given by, [ $1 \times 10000]$.

\subsection{Proposed SoEo algorithm-based deep BiLSTM}

This section portrays the multilingual sentimental analysis module, which is performed using the proposed SoEo algorithm-based BiLSTM using the feature vector established from the input dataset. The architecture of BiLSTM classifier illustrates that the classifier not only saves the historical data but also investigates the input data in two directions (forward and backward), which grooves the significance of texts through considering all the inputs equally, accumulating all the context information and detecting the polarities. Thus, BiLSTM classifier gains significance in the sentiment analysis-related applications, when compared with the traditional recurrent neural networks [26].

\subsubsection{Architecture of deep BiLSTM classifier}

BiLSTM classifier is basically utilized for the sentiment classification of the texts or comments obtained from the tweets or any other social media. The BiLSTM classifier consists of word encoding layer, BiLSTM layer, drop-out layer, and softmax layer, where each layer has unique function in classifying the sentiment. The architecture of BiLSTM is represented in Fig. 2.

i) Word Encoder layer: Word encoding is the representation of words with equal meaning in a unique manner. Consider a sentence $S$ with sequence of tokens as,

$$
T_{i}=\left\{A_{i 1}, A_{i 2}, \ldots, A_{i j}, \ldots A_{i m}\right\}
$$

where, $A_{i j}$ is the $j^{\text {th }}$ word in the $i^{\text {th }}$ sentence and $m$ represents the length of the sentence. The conversion of each word in the sentence to a dimensional vector is known as word embedding. The word embedding matrix $W^{m \times d}$ is constructed after all the words in the sentence are constructed using dimensional vector. Here, $m$ represents the length of the sentence and $d$ represents the embedding size. The parameter of the neural network model is represented by the word embedding matrix and the sentence can be encoded after giving the word embedding matrix as input to the BiLSTM layer.

ii) Bi-LSTM Layer: The extension of the Recurrent Neural Network is termed as the LSTM, which has the capability to resolve the gradient vanishing or exploding problem in standard RNN. The LSTM neural networks are advanced than the standard RNN in architecture, which consist of three gates and a memory cell gate. In this classifier, forward and backward LSTM layers are used to 


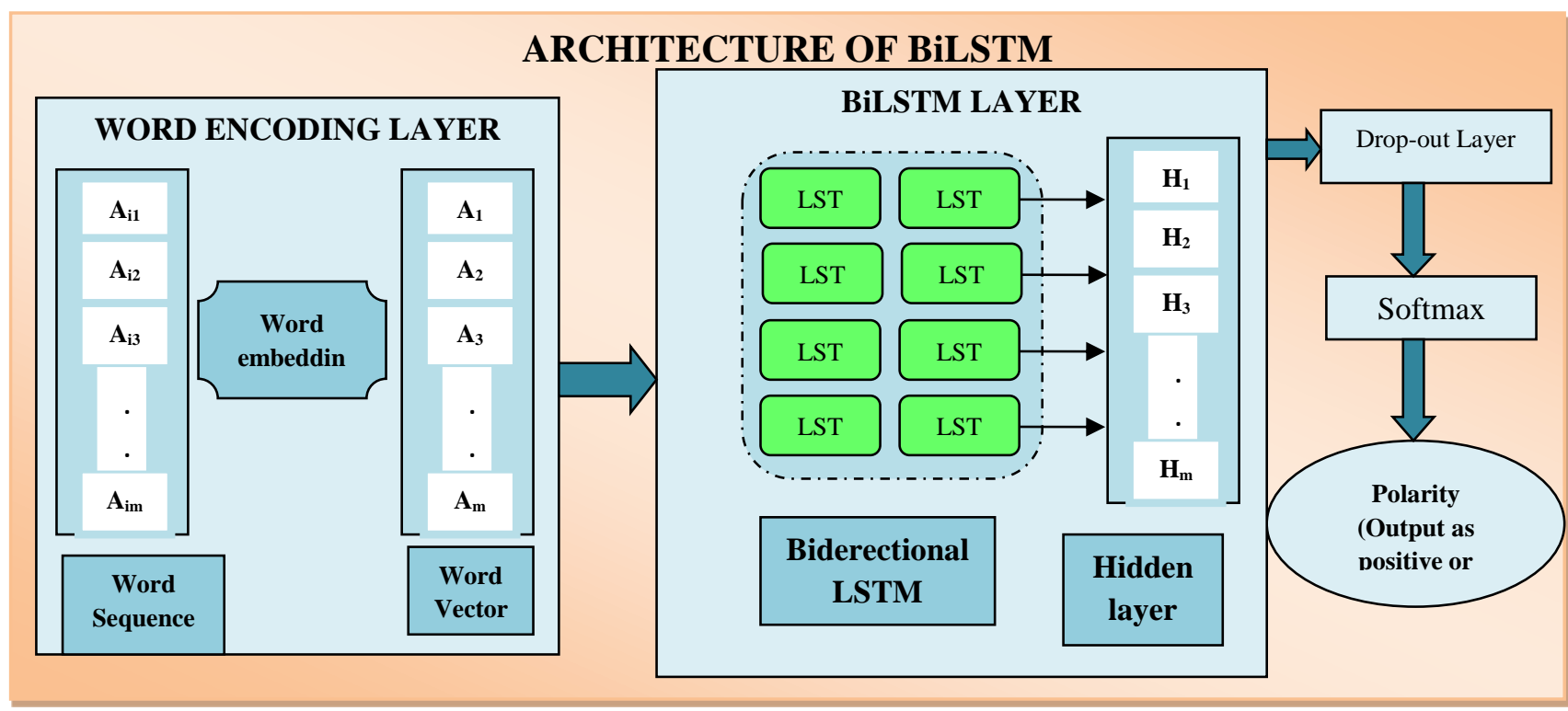

Figure. 2 Architecture of BiLSTM classifier

process the results, where $A=$ $\left\{A_{1}, A_{2}, \ldots \ldots, A_{j}, \ldots, A_{m}\right\}$ represents the word vector in a sentence and $H=$ $\left\{H_{1}, H_{2}, \ldots ., H_{j}, \ldots, H_{m}\right\}$ represents the hidden vector. The single LSTM can be computed as follows:

$$
\begin{gathered}
F_{t}=\sigma\left(w_{f} \cdot C+B_{F}\right) \\
I_{t}=\sigma\left(w_{i}\right) \cdot\left(C+B_{c}\right) \\
O_{t}=\sigma\left|\left(w_{o} \cdot C+B_{o}\right)\right| \\
c_{t}=F_{t} \times c_{t-1}+I_{t} * \tanh \left(w_{c} \cdot C+B_{c}\right) \\
H_{t}=O_{t} \times \tanh \left(c_{t}\right)
\end{gathered}
$$

where, $w_{F}, w_{I}, w_{O}$ represents the weight matrices, $B_{F}, B_{I}, B_{O}$ denotes the biases of the LSTM cells. $\sigma$ represents the sigmoid function and '. ' represents the element wise multiplication. Here, $A_{t}$ and $H_{t}$ represents the word embedding and hidden vector in the sentence $H_{n}$. Forward LSTM evaluates the hidden vector $f H_{t}$ based on $f H_{t-1}$ and the backward LSTM predicts the hidden vector $b H_{t}$ based on $b H_{t-1}$

$$
\begin{aligned}
f H & =\left\{f H_{1}, f H_{2}, \ldots \ldots \ldots, f H_{m}\right\} \\
b H & =\left\{b H_{1}, b H_{2}, \ldots \ldots \ldots . b H_{m}\right\}
\end{aligned}
$$

The output hidden vector $H_{t}$ is given by the product of the forward and backward vectors, which is represented as follows:

$$
H_{t}=\left\{f H_{t}, b H_{t}\right\}
$$

Drop-out layer: The drop-out layer improves the generalization error and enhances the model performance through probabilistically skipping the nodes from activation and weight updation in order to manage the over-fitting issues associated with the deep learning networks. In this research, the dropout rate is fixed to be 0.01 . Dense layer: The output from the LSTM layers is fed to the dense layer, which maps the extracted features in a confined output space of dimension equal to the output class. The dense layer is equipped with two neurons upon which the softmax activation function is applied to derive the probability of the individual class. The output from the BiLSTM model is represented as,

$$
\tilde{G}=\operatorname{soft} \max \left(A_{h} h+J_{h}\right)
$$

The sentence can be represented in a high-level format by multiplying the weights of the word vector $h$ along with the hidden vector and the product is considered as the sentiment feature for sentiment polarity classification. Here $G$ denotes the predicted result through the model, $A_{h}$ represents the weighted matrix and $J_{h}$ stands for bias.

\subsubsection{Proposed SoEo algorithm for hyper-parameter tuning of BiLSTM classifier}

The proposed SoEo algorithm is a meta-heuristic, inspired by the hunting behavior of the bald eagles and the social condition of the coyotes, which exhibits the explorative behavior towards achieving a global optimal solution. Basically, the BES algorithm 
[27] is a nature-inspired meta-heuristic algorithm that reflects the hunting strategy or clever conduct of bald eagles. The advantages of evolutionary and swarm technique are hybridized in the bald eagle optimization algorithm (BES) and the searching pattern of bald eagles outperforms any other optimizations in computational environment. However, during the hunting phase of the bald eagle, the eagles lose energy as the attempt fails for at-least 20 times. Hence, the hunting phase is enhanced through hybridizing the characteristics of coyote [28], which is a nature-inspired algorithm possessing the adaptation behavior with respect to the social condition of the environment. Moreover, the experience characteristics of the coyotes is considered, which is hybridized with the Bald eagle. Thus, hybridizing both the characteristics minimize the error between the empirical data and optical parameter in the selection model. Therefore, the proposed algorithm exhibits the optimal hunting characteristics of the bald eagle and the social condition characteristics of the coyote, which bestow coherent and meticulous effects because the bald eagle always gets focused to lethargic and enervated prey, but the coyote spotlights on sturdy and canny. The process consists of mainly three stages:

i. Hand-pick phase

ii. Scavenge phase

iii. Descending phase

i) Hand-Pick Phase: In this stage, the bald eagle chooses a random space based on the previous information to hunt the prey. The bald eagle always took a place near to the previous search. The modeling of the hand-pick phase is represented as,

$$
R_{\text {new }, k}=R_{\text {best }}+\delta \times s\left(R_{\text {mean }}-R_{k}\right)
$$

where, $\delta$ represents the parameter for controlling the changes in position and $s$ denotes a random number that takes the value between 0 and $1 . R_{\text {best }}$ is the current search space selected by the eagle based on the information from previous search basis and $R_{\text {mean }}$ is the previous information collected by the bald eagle. The current movement of bald eagles is determined by multiplying the randomly searched prior information by $\delta$.

ii) Scavenge Phase: In the search stage, bald eagles search for prey within the selected search space and move in different directions within a spiral space to accelerate their search. The scavenge phase is modelled as,

$$
\begin{aligned}
R_{k, s c a}^{t+1}=R_{k}^{t}+ & i(k)\left(R_{k}^{t}-R_{k+1}^{t}\right) \\
& +j(k)\left(R_{k}^{t}-R_{\text {mean }}^{t}\right)
\end{aligned}
$$

$$
\begin{gathered}
\text { where, } i(k)=\frac{i s(k)}{\max |i s|} \\
j(k)=\frac{j s(k)}{\max (|j s|)} \\
i s(k)=s(i) \times \sin (\theta(k)) \\
j s(k)=s(i) \times \cos (\theta(k)) \\
\theta(k)=b \times \pi \times \text { rand } \\
s(k)=\theta(j)+P \times \text { rand }
\end{gathered}
$$

$b$ is the parameter and it takes the values from 5 to 10 where it determines the corner between point search in the central point. $P$ takes a value between 0.5 and 2 for determining the number of search cycles. $b$ and $P$ notifies the shape when it changes the normal default spiral shape. The polar plot equation to represent the spiral behaviour is mentioned from (iiv). For providing the best solution mean values are used in order to cover intensification and diversification phases. The current social condition can be updated using the social condition and experience characteristics from the coyote optimization algorithm. The detail of the newly obtained model is represented by,

$$
R_{k, \text { coy }}^{t+1}=R_{k}^{t}+\lambda_{1} \partial_{1}+\lambda_{2} \partial_{2}
$$

where, $\partial_{1}$ and $\partial_{2}$ denote the alpha influence and pack influence. The updated equation is given by,

$$
\begin{gathered}
R_{\text {best }}=0.5\left(R_{k, s c a}^{t+1}+R_{k, \text { coy }}^{t+1}\right) \\
R_{\text {best }}=0.5\left[\begin{array}{c}
R_{k}^{t}+i(k)\left(R_{k}-R_{k+1}\right) \\
+j(k)\left(R_{k}-R_{\text {mean }}\right) \\
+R_{k}^{t}+\lambda_{1} \partial_{1}+\lambda_{2} \partial_{2}
\end{array}\right] \\
R_{\text {best }}=0.5\left[\begin{array}{c}
R_{k}^{t}(2+i(k)+j(k))-i(k) R_{k+1}^{t} \\
-j(k) R_{\text {mean }} \\
+\lambda_{1} \partial_{1}+\lambda_{2} \partial_{2}
\end{array}\right]
\end{gathered}
$$

The scavenging phase is promoted using the proposed equation shown in Eq. (24), which increases the exploration phase by enhancing the convergence on to the global solution.

iii) Descend Phase: In the descend stage, bald eagles swing from the best position to the search space to target their prey. But unfortunately, the prey couldn't get spotted at the initial phase. Study shows that one in twenty will be the success rate of hunting the prey by the eagle which in turn causes an energy 
loss during each phase. Since the energy loss of the eagle is proportional to the resting time, the number of phases must be reduced by spotting the prey correctly in order to save the energy. Energy optimization of this eagle is performed by enhancing the descending character by embedding the social condition of coyote. The integrated characteristics of the hunting and social conditioning are performed in a well-organized manner and the convergence rate is greatly improved.

$$
\begin{array}{r}
R_{k, \text { new }}=\text { rand } \times R_{\text {best }}+i(k)\left[R_{\text {mean }}-u_{i}\right] \\
+j(k)\left(R_{\text {best }}-u_{2}\right)
\end{array}
$$

where,

$$
\begin{gathered}
u_{1} \cdot u_{2} \in[1,2] \\
i 1(k)=\frac{i s(k)}{\max |i s|} \\
j 1(k)=\frac{j s(k)}{\max (|j s|)} \\
i s(k)=s(i) \times \sinh (\theta(k)) \\
j s(k)=s(i) \times \cosh (\theta(k))
\end{gathered}
$$

To increase the movement intensity the best solution must be multiplied with random parameters $\mathrm{u} 1$ and $\mathrm{u} 2$. The polar coordinates of the spiral shape are represented in Eqs. (26) to (29). The integrated characteristics of the bald eagle and coyote are performed, and the convergence rate is improved by utilizing the equation below.

$$
\begin{gathered}
R_{k, \text { new }}=0.5 R_{k}^{t}[i(k)-j(k)+1] \\
+0.5\left[\begin{array}{c}
\text { rand } \times R_{\text {best }}+i(k)\left[R_{\text {mean }}-u_{i}\right] \\
+j(k)\left(R_{\text {best }}-u_{2}\right)
\end{array}\right]
\end{gathered}
$$

The bald eagles lose energy in the descend phase because of the number of failure attempts in the preying process. Hence, there is a need for taking rest for the bald eagles to continue preying, which insists the need for enhancing the solution through enhancing the feature with the experience criterions and the social situation of the coyotes. Through the integration of this feature, the bald eagles look for the social condition of the preys while in the descend phase such that the number of the failure attempts of preying is reduced. Thus, the updated equation based on the experience and social condition of the preying is modelled as in Eq. (25).

Algorithmic steps for SoEo algorithm based deep BiLSTM model:

i) Initialization:

Initially, the bald eagles are initialized as, Jand the updation in each phases are repeated for the maximal iterations of $y_{\max }$. The population is denoted as,

$$
R_{k} ;(1 \leq k \leq J)
$$

ii) Determining the fitness functions:

The solution is serviceable in terms of the fitness function such as accuracy, sensitivity and specificity that is given by,

$$
\text { Fitness }=\frac{\text { Accuracy }+ \text { Sensitivity }+ \text { Specificity }}{3}
$$

where, accuracy is the closeness of the true value, sensitivity is the quality of being sensitive, and specificity refers to the ability to correctly identify the parameters. The fitness function should be the maximum of the optimal solution that should boost up the performance. iii) Solution update at different stages in algorithm:

$$
\text { Case1: } R_{\text {new }, k}=R_{\text {best }}+\delta \times s\left(R_{\text {mean }}-R_{k}\right) \text {. }
$$

The first case describes regarding the hand-pick phase, where the area for searching is selected depending upon the previous search information.

$$
\begin{gathered}
\text { Case2: } R_{k}=R_{k}+i(k) \times\left(R_{k}-R_{k+1}\right)+j(k) \times \\
\left(R_{k}-R_{\text {mean }}\right) .
\end{gathered}
$$

The second case is regarding the scavenge phase, where a new area is gleaned from the spiral search and the random number is engendered using axes and movements. When the solution moves towards the centre point or towards the next point, the new point for hunting is guesstimated.

Case3:

$$
\begin{gathered}
R_{k, \text { new }}=\text { rand } \times R_{\text {best }}+i 1(k) \times\left(R_{k}-u 1 \times\right. \\
\left.R_{\text {mean }}\right)+j 1(k) \times\left(R_{k}-u 2 \times R_{\text {best }}\right) .
\end{gathered}
$$

Finally, the descend phases commences, where the descending stage begins when the prey is spotted and then, the suitable best solution is implemented.

iv) Re-evaluation of the Fitness:

The fitness is evaluated for the solutions such that if the fitness of the new solution is better than the fitness of the solution in the previous iteration then, the new solution is retained. In short, the solution that contributes to the maximal value of the fitness is declared as the best solution of the iteration.

v) Termination:

The steps are repeated for the maximal number of the solutions and the best solution is declared. 
Algorithm 1. Pseudo code for proposed SoEo algorithm based deep BiLSTM

\begin{tabular}{|c|c|}
\hline & Proposed SoEo Algorithm \\
\hline & Input: $R_{k} ;(1 \leq k \leq J)$ \\
\hline & Output: $R_{k, \text { new }}$ \\
\hline 1 & Randomly initialize point $\boldsymbol{R}_{\boldsymbol{k}}$ from $\boldsymbol{k}$ point. \\
\hline 2 & Calculate the fitness values of initial point $R_{k}$ \\
\hline 3 & $\begin{array}{l}\text { While }\left(y=y_{\max }\right) \text { (Termination conditions are } \\
\text { not met) }\end{array}$ \\
\hline & \#Hand-pick space \\
\hline 4 & For(each point $k$ in the population) \\
\hline 5 & $s\left(R_{\text {mean }}-R_{k}\right) \quad R_{\text {new }, k}=R_{\text {best }}+\delta \times$ \\
\hline 6 & iff $\left(R_{\text {new }}\right)<f\left(R_{k}\right)$ \\
\hline 7 & $R_{k}=R_{\text {new }}$ \\
\hline 8 & iff $\left(R_{\text {new }}\right)<f\left(R_{\text {best }}\right)$ \\
\hline 9 & $R_{\text {best }}=R_{\text {new }}$ \\
\hline 10 & End if \\
\hline 11 & End if \\
\hline 12 & End for \\
\hline & \#Search in space \\
\hline 13 & For(each point $k$ in the sentence) \\
\hline 14 & $\begin{array}{r}R_{\text {best }}=0.5\left[R_{k}^{t}(2+i(k)+j(k))-i(k) R_{k+1}^{t}\right. \\
\left.-j(k) R_{\text {mean }}+\lambda_{1} \partial_{1}+\lambda_{2} \partial_{2}\right]\end{array}$ \\
\hline 15 & iff $\left(R_{\text {best }}\right)<f\left(R_{k}\right)$ \\
\hline 16 & $R_{k}=R_{\text {best }}$ \\
\hline 17 & iff $\left(R_{\text {new }}\right)<f\left(R_{\text {best }}\right)$ \\
\hline 18 & $R_{\text {best }}=R_{\text {new }}$ \\
\hline 19 & End if \\
\hline 20 & End if \\
\hline 21 & End for \\
\hline & \# Descend Phase \\
\hline 22 & For(each point $k$ in the sentence) \\
\hline 23 & $\begin{array}{c}R_{k, \text { new }}=\text { rand } \times R_{\text {best }}+i 1(k) \times\left(R_{k}-\right. \\
\left.u 1 \times R_{\text {mean }}\right)+j 1(k) \times\left(R_{k}-u 2 \times R_{\text {best }}\right)\end{array}$ \\
\hline 24 & iff $\left(R_{\text {new }}\right)<f\left(R_{k}\right)$ \\
\hline 25 & $R_{k}=R_{\text {new }}$ \\
\hline 26 & if $f\left(R_{\text {new }}\right)<f\left(R_{\text {best }}\right)$ \\
\hline 27 & $R_{\text {best }}=R_{\text {new }}$ \\
\hline 28 & End if \\
\hline 29 & End if \\
\hline 30 & End for \\
\hline 31 & Assigny $=y+1$ \\
\hline 32 & END WHILE \\
\hline
\end{tabular}

\section{Result and discussion}

The sentiment present in the comments is analyzed in order to predict the polarity of the model so that the promotion of a product or growth of organization can be achieved. The proposed SoEo deep-BiLSTM is implemented using Sentiment 140 dataset and Twitter Sentiment Analysis Dataset. The experimental results are enumerated in the following section in order to prove their efficiency.

\subsection{Experimental setup}

The experiment is implemented in Python and Pycharm and the system configuration of the experiment includes Python 3.7.6 and Pycharm 2020Community Edition running in Windows 10 operating system with 8 GB RAM memory.

\subsection{Performance metrics}

The performance metrics is used to analyze the performance of the proposed model in the sentiment classification using the multilingual dataset.

Accuracy: The accuracy can be defined as the state of being correct in the case of classification, which in other words is defined as the ratio of the sum of total true positives and true negatives to the sum of all total positive and negative samples as given by,

$$
\text { Accuracy }=\frac{T_{p o s}+T_{n e g}}{T_{p o s}+T_{n e g}+F_{p o s}+F_{n e g}}
$$

Precision: The percentage of the relevant instances or true positives to the total positives is referred as precision, which is given by,

$$
\operatorname{Pr} \text { e cision }=\frac{T_{\text {pos }}}{T_{p o s}+F_{p o s}}
$$

Recall: Recall is the ratio of the total positives to the sum of the total positives and negatives as given by,

$$
\text { Re c all }=\frac{T_{\text {pos }}}{T_{\text {pos }}+F_{\text {neg }}}
$$

F1 Measure: The F1 measure is defined as the harmonic mean of the precision and sensitivity as given by,

$$
\begin{array}{r}
F 1 \text { measure }=(2 \times \text { Pr e cision } \times \text { recall }) \\
/(\text { Pr e cision }+ \text { recall })
\end{array}
$$

\subsection{Performance analysis}

\subsubsection{Performance analysis using sentiment 140 dataset}

Fig. 3 illustrates the performance analysis of the proposed SoEo deep-BiLSTM in terms of accuracy, precision, recall, and F1 measure by varying the epochs. The Proposed SoEo deep-BiLSTM performs better in the accuracy rate when the training dataset of varying epoch $20,40,60,80,100$ are provided which can be proved by providing the sample values obtained when the epoch is at 100 the values are $79.020 \%, 82.872 \%, 86.148 \%, 88.196 \%, 96.687 \%$ respectively. Similarly, when it trained with $90 \%$ of 


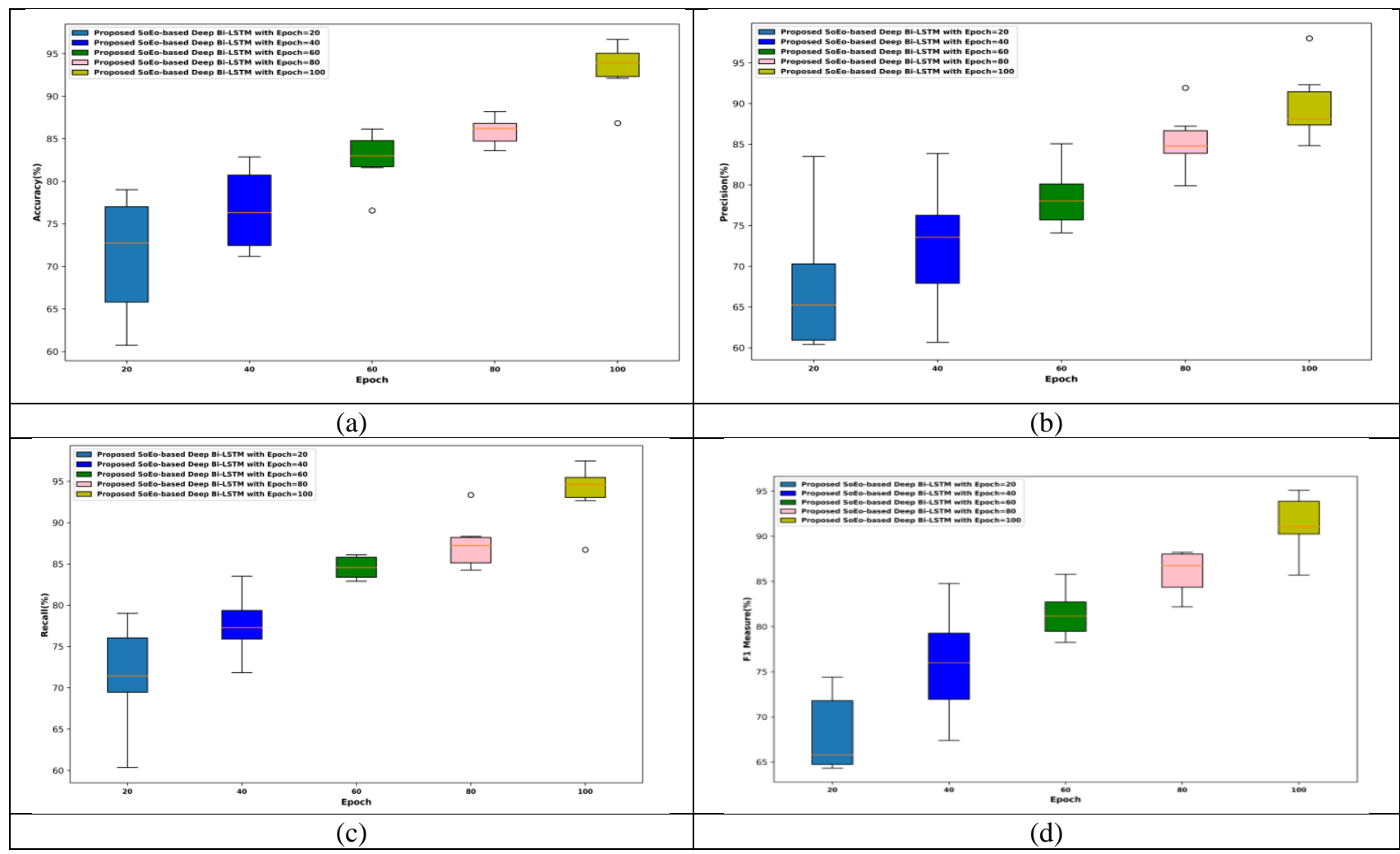

Figure. 3 Parameters for analyzing the performance of proposed SoEo deep-BiLSTM using Sentiment 140 dataset: (a) Accuracy, (b) Precision, (c) Recall, and (d) F1 measure

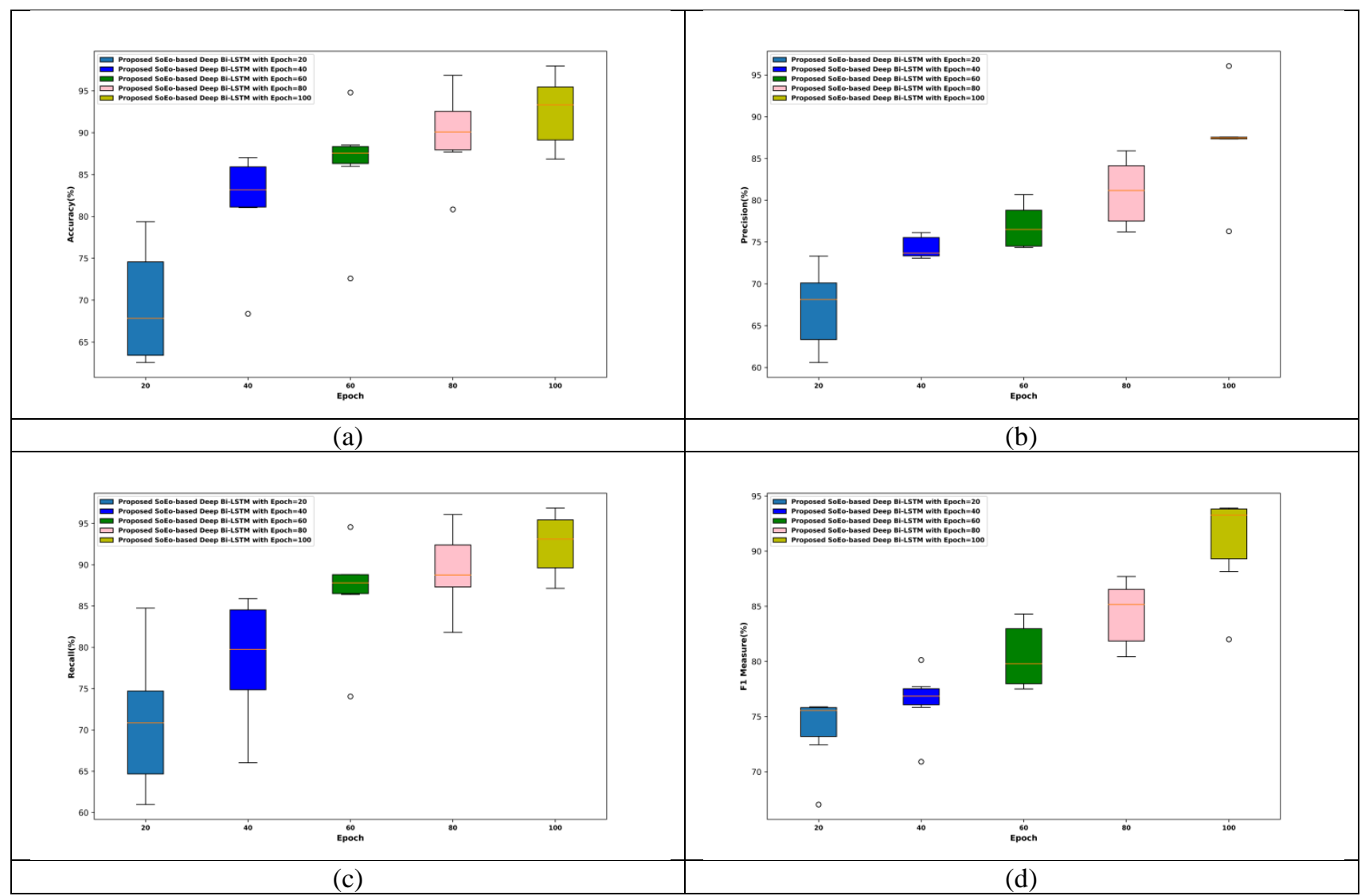

Figure. 4 Parameters for analyzing the performance of proposed SoEo deep-BiLSTM using twitter sentiment analysis dataset: (a) Accuracy, (b) Precision, (c) Recall, and (d) F1 measure 
training data the values obtained for precision are $83.486 \%, 83.878 \%, 85.060 \%, 91.920 \%, 98.000 \%$ respectively. Likewise, the recall evaluated for the epoch values are $79.016 \%, 83.488 \%, 86.109 \%$, $93.341 \%, 97.4592 \%$ respectively. Eventually the F1 is measured for the aforementioned epoch value is enumerated as follows $74.396 \%, 84.777 \%$, $85.809 \%, 88.234 \%$ and $95.107 \%$ respectively.

\subsubsection{Performance analysis using twitter sentiment analysis dataset}

Fig. 4 illustrates the performance analysis of the proposed SoEo deep-BiLSTM in terms of accuracy, precision, recall and $\mathrm{F} 1$ measure by varying the epochs. The analysis of the proposed SoEo deepBiLSTM in terms of the accuracy by varying the epoch $20,40,60,80$, and 100 with $80 \%$ of training data are $76.158 \%, 86.206 \%, 88.523 \%, 92.932 \%$, $95.670 \%$ respectively, which is depicted in Fig. 4 (a). Similarly, the precision evaluated by the proposed method with $60 \%$ of training data by varying the epoch $20,40,60,80$, and 100 is $69.405 \%, 74.002 \%$, $78.482 \%, 82.052 \%, 87.517 \%$ respectively, which is illustrated in Fig. 4 (b). Likewise, the recall evaluated by the proposed SoEo deep-BiLSTM with $80 \%$ of training data with the epoch $20,40,60,80$, and 100 is $84.76 \%, 85.91 \%, 94.57 \%, 96.08 \%, 96.87 \%$ respectively, which is shown in Fig. 4 (c). Finally, the F1 measure for the various epoch values $20,40,60$, 80,100 is $75.88 \%, 80.13 \%, 84.30 \%, 87.70 \%$, $93.91 \%$ respectively shown in Fig. 4 (d).

\subsection{Comparative methods}

The methods used for the comparison includes Naïve Bayes [29], XG Boost [30], Random Forest [31], Decision tree [32], Valance based Lexicon model [33], Deep BiLSTM [34], Coyote based deep BiLSTM [35], BES based deep BiLSTM [36], which are compared with the proposed method. These existing machine learning classifiers are employed for the comparative analysis through the comparative discussion of the proposed method with the existing methods based on the performance measures to justify the effectiveness of the proposed method. The proposed classifier is the hybrid approach of BES and coyote and hence, the comparison shows the results of BES and coyote along with the machine learning models, like Naïve bayes, decision tree, random forest, and lexicon approach against the deep BiLSTM classifier.

\subsection{Comparative analysis}

In this analysis the comparison is made using the sentiment 140 dataset and twitter sentiment analysis dataset where the parameters accuracy, precision, recall, and f1 measures are considered to reveal the importance of the proposed method.

\subsubsection{Comparative analysis using the sentiment 140 dataset}

Fig. (5) shows the comparative analysis of the methods using sentiment 140 dataset. The accuracy, precision, recall, and $\mathrm{F} 1$ measures are depicted in the Fig. 5(a), 5(b), 5(c), and 5(d) respectively. The accuracy rate of methods such as Naïve Bayes, XG Boost, Random Forest, Decision tree, Valance based Lexicon model, Deep BiLSTM, Coyote based deep BiLSTM, BES based deep BiLSTM and proposed SoEo deep-BiLSTM is $72.394 \%, 75.214 \%$, $78.631 \%, 84.351 \%, 84.904 \%, 86.415 \%, 88.147 \%$, $88.474 \%$, and $91.265 \%$ respectively when the training percentage is $90 \%$. Similarly for the precision, the percentage of the methods Naïve Bayes, XG Boost, Random Forest, Decision tree, Valance based Lexicon model, Deep BiLSTM, Coyote based deep BiLSTM, BES based deep BiLSTM and proposed SoEo deep-BiLSTM is $68.153 \%, 70.086 \%$, $76.129 \%, 78.585 \%, 85.004 \%, 86.179 \%, 87.923 \%$, $90.147 \%$ and $90.400 \%$ respectively. Furthermore, the recall percentage of the methods Naïve Bayes, XG Boost, Random Forest, Decision tree, Valance based Lexicon model, Deep BiLSTM, Coyote based deep BiLSTM, BES based deep BiLSTM is $67.594 \%$, $76.638 \%, 78.332 \%, 82.232 \%, 87.162 \%, 87.558 \%$, $88.298 \%, 88.917 \%, 91.780 \%$ for $90 \%$ of training. At last the F1 measure of the training dataset corresponding to the methods Naïve Bayes, XG Boost, Random Forest, Decision tree, Valance based Lexicon model, Deep BiLSTM, Coyote based deep BiLSTM, BES based deep BiLSTM and proposed SoEo deep-BiLSTM for $90 \%$ of training set is listed as $65.188 \%, 71.091 \%, 75.473 \%, 77.265 \%$, $82.240 \%, 85.391 \%, 88.833 \%, 89.535 \%$ and $90.871 \%$ respectively.

\subsubsection{Comparative analysis using twitter sentiment analysis dataset}

Fig. 6 shows the performance analysis of the methods using Twitter sentiment analysis dataset. The accuracy rate of the methods Naïve Bayes, XG Boost, Random Forest, Decision tree, Valance based Lexicon model, Deep BiLSTM, Coyote based deep BiLSTM, BES based deep BiLSTM and proposed SoEo deep-BiLSTM and proposed SoEo deep- 


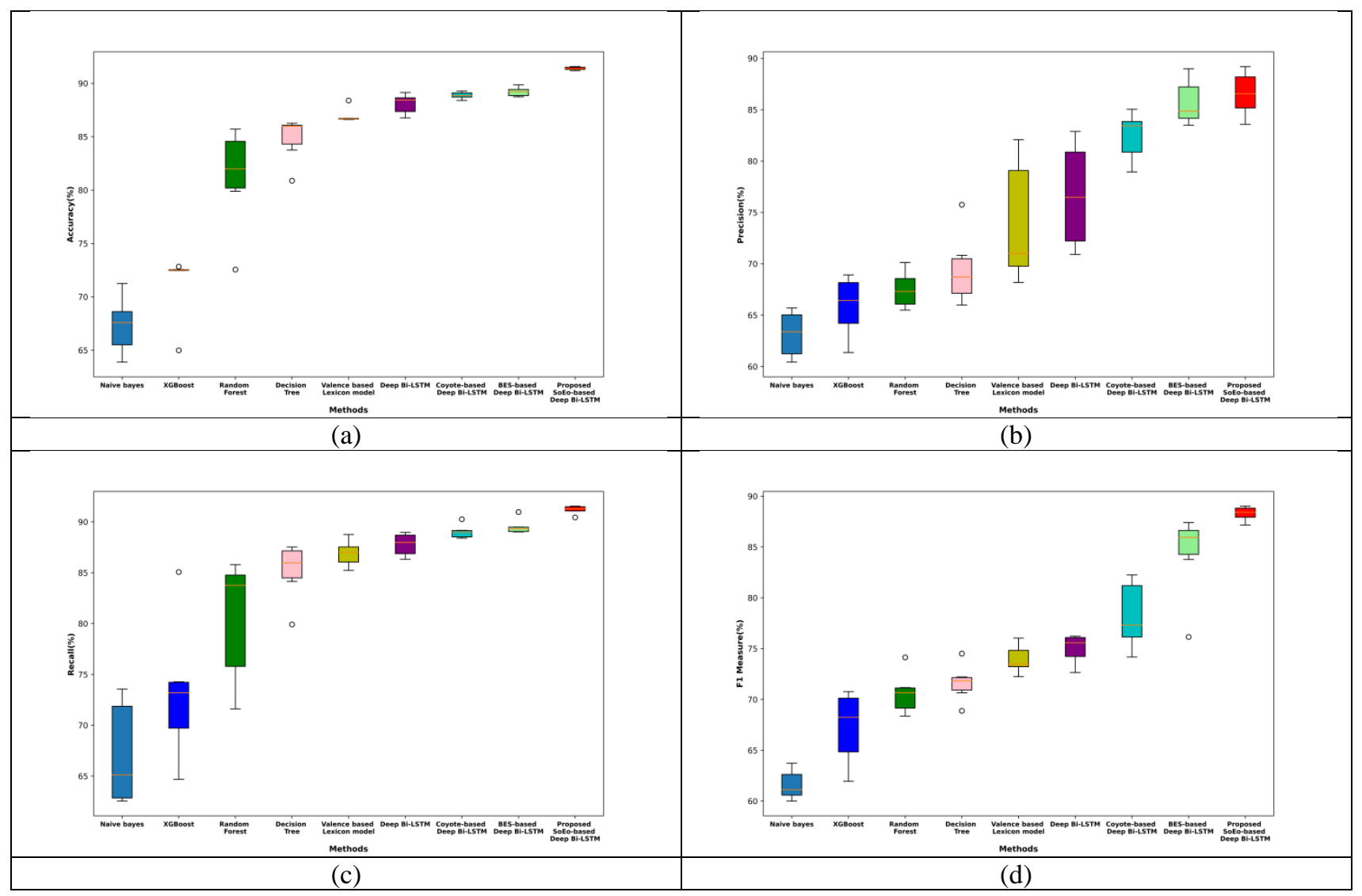

Figure. 6 Parameters to compare the proposed SoEo deep-BiLSTM using twitter sentiment analysis dataset: (a) Accuracy, (b) Precision, (c) Recall, and (d) F1 measure

BiLSTM is given as $71.251 \%, 72.836 \%, 85.712 \%$, $86.260 \%, 88.387 \%, 89.143 \%, 89.268 \%, 89.856 \%$ and $91.572 \%$ for $90 \%$ of training data. Correspondingly the precision rate of the Naïve Bayes, XG Boost, Random Forest, Decision tree, Valance based Lexicon model, Deep BiLSTM, Coyote based deep BiLSTM, BES based deep BiLSTM methods and proposed SoEo deep-BiLSTM is given by $65.699 \%, 68.917 \%, 70.132 \%, 75.743 \%$, $82.083 \%, 82.883 \%, 85.031 \%, 88.971 \%, 89.196 \%$ respectively. Comparably the recall rate of the methods Naïve Bayes, XG Boost, Random Forest, Decision tree, Valance based Lexicon model, Deep BiLSTM, Coyote based deep BiLSTM, BES based deep BiLSTM and proposed SoEo deep-BiLSTM is given by $73.559 \%, 85.062 \%, 85.798 \%, 87.526 \%$, $88.758 \%, 88.962 \%, 90.259 \%, 90.967 \%$ respectively. Finally, the f1 measure is given as $63.721 \%, 70.770 \%, 74.130 \%, 74.505 \%, 76.033 \%$, $76.221 \%, 82.249 \%, 87.402 \%$ and $89.019 \%$ for the methods Naïve Bayes, XG Boost, Random Forest, Decision tree, Valance based Lexicon model, Deep BiLSTM, Coyote based deep BiLSTM, BES based deep BiLSTM and proposed SoEo deep-BiLSTM.

\subsection{Comparative discussion}

This section deliberates the methods employed for the prediction of sentiment based on the tweets or comments. The methods utilized for the comparison are Naïve Bayes, XG Boost, Random Forest, Decision tree, Valance based Lexicon model, Deep BiLSTM, Coyote based deep BiLSTM, BES based deep BiLSTM which are analysed from the basic level of the sentiment prediction. The parameters used for the proposed method are assigned from the dataset of sentiment 140 dataset and Twitter sentiment analysis dataset. While the training is performed for all the methods the results shows that the parameters such as accuracy, precision, recall and F1 measure are increased leads to the greater improvement in the prediction of the sentiment.

\section{Conclusion}

The research on the BiLSTM classifier for sentiment prediction utilizing the available data is implemented and analyzed in this paper. The significance of the project is based on the proposed meta-heuristic nature inspired algorithm named SoEo algorithm which predicts the sentiment based on the 
Table 1. Comparative discussion

\begin{tabular}{|c|c|c|c|c|c|c|c|c|}
\hline \multirow{2}{*}{ Methods } & \multicolumn{5}{|c|}{ Sentiment 140 dataset } & \multicolumn{3}{c|}{ Twitter sentiment analysis dataset } \\
\cline { 2 - 9 } & $\begin{array}{c}\text { Accuracy } \\
(\%)\end{array}$ & $\begin{array}{c}\text { Precision } \\
(\%)\end{array}$ & $\begin{array}{c}\text { Recall } \\
(\%)\end{array}$ & $\begin{array}{c}\text { F1 } \\
\text { Measure } \\
(\%)\end{array}$ & $\begin{array}{c}\text { Accuracy } \\
(\%)\end{array}$ & $\begin{array}{c}\text { Precision } \\
(\%)\end{array}$ & $\begin{array}{c}\text { Recall } \\
(\%)\end{array}$ & $\begin{array}{c}\text { F1 } \\
\text { Measure } \\
(\%)\end{array}$ \\
\hline $\begin{array}{c}\text { Naïve } \\
\text { Bayes }\end{array}$ & 65.188 & 72.394 & 68.153 & 67.594 & 71.251 & 65.699 & 73.559 & 63.721 \\
\hline XG Boost & 71.091 & 75.214 & 70.086 & 76.638 & 72.836 & 68.917 & 85.062 & 70.770 \\
\hline $\begin{array}{c}\text { Random } \\
\text { Forest }\end{array}$ & 75.473 & 78.631 & 76.129 & 78.332 & 85.712 & 70.132 & 85.798 & 74.130 \\
\hline $\begin{array}{c}\text { Decision } \\
\text { tree }\end{array}$ & 77.265 & 84.351 & 78.585 & 82.232 & 86.260 & 75.743 & 87.526 & 74.505 \\
\hline $\begin{array}{c}\text { Valance } \\
\text { based } \\
\text { Lexicon } \\
\text { model }\end{array}$ & 82.24 & 84.904 & 85.004 & 87.162 & 88.387 & 82.083 & 88.758 & 76.033 \\
\hline $\begin{array}{c}\text { Deep } \\
\text { BiLSTM }\end{array}$ & 85.391 & 86.415 & 86.179 & 87.558 & 89.143 & 82.883 & 88.962 & 76.221 \\
\hline $\begin{array}{c}\text { Coyote } \\
\text { based deep } \\
\text { BiLSTM }\end{array}$ & 88.833 & 88.147 & 87.923 & 88.298 & 89.268 & 85.031 & 90.259 & 82.249 \\
\hline $\begin{array}{c}\text { BES based } \\
\text { deep } \\
\text { BiLSTM }\end{array}$ & 89.535 & 88.474 & 90.147 & 88.917 & 89.856 & 88.971 & 90.967 & 87.402 \\
\hline $\begin{array}{c}\text { Proposed } \\
\text { SoEo } \\
\text { deep- } \\
\text { BiLSTM }\end{array}$ & 91.265 & 90.400 & 91.780 & 90.871 & 91.572 & 89.196 & 91.551 & 89.019 \\
\hline
\end{tabular}

polarity of the data. The classifier train and classify the data in bidirectional is a add on benefit to the research which utilizes all the information's given on the data and the performance is compared with various other standard classification models. The analysis of the sentiment is carried through the classifier using the sentiment 140 dataset and Twitter sentiment analysis dataset and the performance metrics accuracy, precision, recall and F1 measure are examined. The performance characteristics shows that the proposed model obtained an accuracy of $91.572 \%$, precision of $89.196 \%$, recall of $91.551 \%$ and F1 measure of $89.019 \%$ which will be more efficient compared to the state-of-art methods.

\section{Conflicts of Interest}

The authors declare no conflict of interest.

\section{Author Contributions}

Conceptualization of research topic is done by the author-1 and after review by author 2 the methodology is finalized by author 1 . Author 1 implemented the methodology using python. Validation is performed by doing formal analysis by both the authors. The investigation into results is carried out by the author 2. Data curation, draft preparation, review, and editing is done by author 1 . Author 2 carried out supervision.

\section{References}

[1] R. Bhargava and Y. Sharma, "MSATS: Multilingual sentiment analysis via text summarization", Data Science \& EngineeringConfluence, IEEE, pp. 71-76, 2017.

[2] B. Liu, "Opinion mining and sentiment analysis", Web Data Mining, pp. 459-526, 2011.

[3] L. R. C. Pessutto, D. S. Vargas, and V. P. Moreira, "Multilingual aspect clustering for sentiment analysis", Knowledge-Based Systems, Vol. 192, p. 105339, 2020.

[4] Y. Madani, M. Erritali, and J. Bengourram, "A Hybrid Multilingual Sentiment Classification Using Fuzzy Logic and Semantic Similarity", In: Proc. of International Conference on Advanced Intelligent Systems for Sustainable Development, pp. 145-158, 2018.

[5] E. Cambria, D. Das, S. Bandyopadhyay, and A. Feraco, "Affective computing and sentiment analysis", A Practical Guide to Sentiment Analysis, pp. 1-10, 2017. 
[6] M. Graff, S. M. Jimenez, E. S. Tellez, and D. Moctezuma, "Evomsa: A multilingual evolutionary approach for sentiment analysis [application notes]", IEEE Computational Intelligence Magazine, Vol. 15, No. 1, pp.76-88, 2020.

[7] A. K. Soni, "Multi-lingual sentiment analysis of Twitter data by using classification algorithms", In: Proc. of IEEE Second International Conference on Electrical, Computer and Communication Technologies, pp. 1-5, 2017.

[8] Y. Madani, M. Erritali, J. Bengourram, and F. Sailhan, "A multilingual fuzzy approach for classifying Twitter data using fuzzy logic and semantic similarity", Neural Computing and Applications, Vol. 32, No. 12, pp. 8655-8673, 2020.

[9] C. R. Banea, Mihalcea, and J. Wiebe, "Multilingual subjectivity: Are more languages better?", In: Proc. of the International Conference on Computational Linguistics, pp. 28-36, 2010.

[10] S. L. Lo, E. Cambria, R. Chiong, and D. Cornforth, "Multilingual sentiment analysis: From formal to informal and scarce resource languages", Artificial Intelligence Review, Vol. 48, No. 4, pp. 499-527, 2017.

[11] R. Steinberger, "A survey of methods to easy the development of highly multilingual text mining applications", Language Resources and Evaluation, Vol. 46, pp. 155-176, 2012.

[12] D. Vilares, H. Peng, R. Satapathy, and E. Cambria, "BabelSenticNet: a commonsense reasoning framework for multilingual sentiment analysis", In: Proc. of 2018 IEEE Symposium Serieson Computational Intelligence, pp. 1292$1298,2018$.

[13] M. Thelwall, K. Buckley, and G. Paltoglou, "Sentiment strength detection for the social web", Journal of the American Society for Information Science and Technology, Vol. 63, No. 1, pp. 163-173, 2012.

[14] D. Vilares, M. Thelwall, and M. A. Alonso, "The megaphone of the people? spanish sentistrength for real-time analysis of political tweets", Journal of Information Science, Vol. 41, No. 6, pp. 799-813, 2015.

[15] A. Hogenboom, B. Heerschop, F. Frasincar, U. Kaymak, and F. D. Jong, "Multi-lingual support for lexicon-based sentiment analysis guided by semantics", Decision Support Systems, Vol. 62, pp. 43-53, 2014.

[16] H. Ghorbel and D. Jacot, "Sentiment analysis of french movie reviews", Advances in Distributed Agent-Based Retrieval Tools, ser. Studies in
Computational Intelligence, Vol. 361, pp. 97108, 2011.

[17] J. Wehrmann, W. Becker, H. E. Cagnini, and R. C. Barros, "A character-based convolutional neural network for language-agnostic Twitter sentiment analysis", International Joint Conference on Neural Networks, pp. 2384-2391, 2017.

[18] V. K. Jain, S. Kumar, and S. L. Fernandes, "Extraction of emotions from multilingual text using intelligent text processing and computational linguistics", Journal of Computational Science, Vol. 21, pp. 316-326, 2017.

[19] T. Rao, X. Li, H. Zhang, and M. Xu, "Multilevel region-based convolutional neural network for image emotion classification", Neurocomputing, Vol. 333, pp. 429-439, 2019.

[20] L. Sun, B. Zou, S. Fu, J. Chen, and F. Wang, "Speech emotion recognition based on DNNdecision tree SVM model", Speech Communication, Vol. 115, pp. 29-37, 2019.

[21] W. Zehra, A. R. Javed, Z. Jalil, H. U. Khan, and T. R. Gadekallu, "Cross corpus multi-lingual speech emotion recognition using ensemble learning", Complex \& Intelligent Systems, pp. 110, 2021.

[22] X. Li, M. Akagi, "Improving multilingual speech emotion recognition by combining acoustic features in a three-layer model", Speech Communication, Vol. 110, pp. 1-12, 2019.

[23] T. T. Sasidhar, B. Premjith, and K. P. Soman, "Emotion detection in Hinglish (Hindi+ English) code-mixed social media text", Procedia Computer Science, Vol. 171, pp. 13461352, 2020.

[24] https://www.kaggle.com/kazanova/sentiment14 0

[25] https://github.com/sharmaroshan/Twitter-Senti ment-Analysis

[26] G. Xu, Y. Meng, X. Qiu, Z. Yu, and X. Wu, "Sentiment Analysis of Comment Texts Based on BiLSTM", IEEE Access, Vol. 7, pp. 5152251532, 2019.

[27] H. A. Alsattar, A. A. Zaidan, and B. B. Zaidan, "Novel meta-heuristic bald eagle search optimisation algorithm", Artificial Intelligence Review, Vol. 53, No. 3, pp. 2237-2264, 2020.

[28] J. Pierezan and L. D. S. Coelho, "Coyote optimization algorithm: a new metaheuristic for global optimization problems", In: Proc. of 2018 IEEE Congress on Evolutionary Computation, pp. 1-8, 2018.

[29] I. Rish, "An empirical study of the naive Bayes classifier", IJCAI 2001 Workshop on Empirical 
Methods in Artificial Intelligence, Vol. 3, No. 22, pp. 41-46, 2001.

[30] T. Chen and C. Guestrin, "Xgboost: A scalable tree boosting system", In: Proc. of the 22nd Acm Sigkdd International Conference on Knowledge Discovery and Data Mining, pp. 785-794, 2016.

[31] M. Pal, "Random Forest classifier for remote sensing classification", International Journal of Remote Sensing 26, No. 1, pp. 217-222, 2005.

[32] J. R. Quinlan, "Learning decision tree classifiers", ACM Computing Surveys 28, No. 1, pp. 71-72, 1996.

[33] A. Masum, S. Mostafa, H. Prendinger, and M. Ishizuka, "SenseNet: A linguistic tool to visualize numerical-valence based sentiment of textual data", In: Proc. of the International Conference on Natural Language Processing, pp. 147-152, 2007.

[34] M. Sajjad and S. Kwon, "Clustering-based speech emotion recognition by incorporating learned features and deep BiLSTM", IEEE Access 8, pp. 79861-79875, 2020.

[35] D. Dangi, A. Bhagat, and D. K. Dixit, "Sentiment analysis of social media data based on chaotic coyote optimization algorithm-based time weight-AdaBoost support vector machine approach", Concurrency and Computation: Practice and Experience, 2021.

[36] J. Shobana and M. Murali, "Adaptive particle swarm optimization algorithm based long shortterm memory networks for sentiment analysis", Journal of Intelligent \& Fuzzy Systems Preprint, pp. 1-17, 2021. 